\title{
Les pulsations du champ magnétique terrestre et des courants telluriques
}

(Description des Phénomènes)

J. COULOMB

ricevuto il 1 settembre 1959

\section{INTRODUCTION}

L'étude des pulsations magnétiques connaît depuis quelques années un renouveau étonnant, du côté instrumental aussi bien que du coté théorique (où se développent des idées, issues de celles de Dungey, sur les oscillations de l'exosphère). Pour permettre aux géophysiciens intéressés d'aborder avec fruit les discussions publiées de divers côtés, il a paru utile de faire le point des résultats d'observation. L'article qui suit ne prétend pas à plus d'originalité. Il a été écrit à la suite d'un séminaire tenu à l'Institut de Physique du Globe de Paris et il utilise certains des exposés qui ont été faits à ce séminaire. Il suppose connue la liaison générale entre phénomènes géomagnétiques et telluriques simultanés (voir par exemple L. Cagniard, Electricité tellurique, Handbuch der Physik, Bd XLVII, Geophysik I, pp. 407-469).

\section{GÉNÉRALites: $p t, p c$.}

Le nom de pulsations donné en 1899 par Van Bemmelen s'appliquait à des phénomènes visibles parfois sur les enregistrements magnétiques relativement lents, c'est à dire à ce qu'on appelle aujourd'hui des $p t$ (pulsation trains, trains de pulsation) avec la définition suivante, due au "Comité des variations magnétiques rapides et des courants telluriques" nommé par l'Association Internationale de Géomagnétisme et d'Aéronomie (1): "Séries d'oscillations, chaque série durant en général de 10 à 20 minutes, l'ensemble du phénomène ne dépassant guère une heure ". La période des oscillations, qui est en général comprise entre 30 et $100 \mathrm{sec}$, peut atteindre parfois plusieurs minutes; leur amplitude qui est en général de l'ordre du gamma $\left(1 \gamma=10^{-5} 0 e\right)$ sur les composantes horizontales peut exceptionnellement atteindre ou dépasser $10 \gamma$. O'est ce qui explique 
qu'elles pouvaient apparaître sur les enregistrements anciens. La composante verticale est en général (*) beaucoup plus faible, ce qui est naturel pour les variations observées à la surface d'un conducteur.

Il est naturel d'étendre le mot de pulsations à des oscillations d'amplitude mojenne plus faible (de l'ordre de $0,1 \gamma$ sur les composantes horizontales), prolongées, régulières, bien décrites par Eschenhagen dès 1896; on les appelle aujourd'hui des pc (pulsations continues) avec pour définition officielle: "Pulsations présentant une continuité notable, d'une période comprise entre 10 et 40 secondes (exceptiomellement jusqu'à $50 \mathrm{sec}$ ) et durant plusieurs heures n. Eschenhagen appelait ces oscillations des "ondes élémentaires " parce qu'il était persuadé, à tort d'ailleurs, qu'on n'obtiendrait jamais de détails plus fins.

Certains auteurs appellent pulsations non seulement ces deux espèces de variations assez régulières, mais n'importe quelle agitation serrée dans laquelle l'intervalle de deux maximums successifs (la période comme on dit par abus de langage commode) soit inférieure à quelques minutes par exemple. C'est ainsi qu'on a attribué à Balfour Stewart le mérite d'avoir observé les pulsations pour la première fois. En fait il décrit la grande tempête magnétique de 1859 au cours de laquelle Carrington fit, en lumière blanche, la première observation d'éruption chromosphérique. Cette tempête accompagnait une aurore boréale visible en Angleterre et comportait l'agitation désordonnée habituelle en pareil cas; Balfour Stewart put $\mathrm{y}$ distinguer des périodes relativement courtes (de $1 / 2$ à 5 minutes).

Les indications précédentes s'appliquent mal aux régions polaires. Nous décrirons plus loin certains phénomènes qui leur sont particuliers. Bornons nous pour l'instant aux régions de latitude moyenne ou basse, et considérons une journée où les enregistrements magnétiques lents soient un peu agités. Les enregistrements magnétiques rapides montrent alors presque toujours de nombreuses pulsations. Leur amplitude atteint quelques $\gamma$ sur les composantes horizontales. Mais, comme l'avait déjà vu Eschenhagen, leur aspect est très différent de jour où dominent les $p c$ et de nuit où les $p t$ les remplacent $\left({ }^{* *}\right)$. La distinction apparaît à pre-

(*) Les stations côtières (Dumont d'Urville) peuvent faire exception.

$\left.{ }^{* *}\right) 11$ faut cependant signaler que Mme Troutzkaia a énoncé en $1953\left(^{3}\right)$, et semble avoir maintenu depuis, l'idée que la séparation des $p c$ et des $p t$ serait réglée non pas d'après le temps local mais d'après le temps universel. Les $p c$ auraient lieu de $0 \mathrm{~h}$ à $12 \mathrm{~h}$ TU, le plus souvent de $2 \mathrm{~h}$ à $4 \mathrm{~h}$. Les $p t$ auraient lieu de $12 \mathrm{~h}$ à $24 \mathrm{~h}$ TU le plus souvent de $18 \mathrm{~h}$ à $19 \mathrm{~h}$. Les observations 
mière vue sur les enregistrements (fig. 1) et peut être aisément confirmée par la statistique. C'est ainsi que Holmberg $\left({ }^{2}\right)$ trouve que les périodes à Eskdalemuir se groupent autour des deux valeurs $25 \mathrm{sec}$ et $70 \mathrm{sec}$ (en moyenne annuelle). Les heures proches de minuit sont calmes. Les premières pulsations, de période voisine de $30 \mathrm{sec}$, sont très faibles. Puis leur amplitude croît et les deux types se séparent vers l'heure du lever du soleil. Au cours de l'après-midi, la période de la bande lente augmente; au coucher du soleil les pulsations correspondantes prennent la forme de trains $p t$ caractéristiques. Toujours selon Holmberg, la séparation des bandes se produirait plus tot en été qu'en hiver. Ceci n'est pas en contradiction avec une statisque des $p c$ à Chambon-la-Forêt (par G. Gibault, non publiée), peu nette à vrai dire. En Terre-Adélie, c'est le contraire qui paraît vrai.

Phénomìnes nocturnes Des LATITUdes MoYennes: $p t$ ACCOMPaGNANT LES BAIES.

On ne voit la nuit que quelques $p t$, accompagnant souvent certaines variations amples et lentes des éléments magnétiques, qu'on appelle des baies magnétiques parce qu'elles comportent un aller-retour sur l'une des composantes (fig. 1).

L'association des deux phénomènes a été reconnue depuis longteinps, notamment par Terada en 1917 et G. Angenleister en $1920\left(^{4}\right)$. Comıne Hatakeyama l'a noté en $1938\left({ }^{5}\right)$, Giacomo en $1940\left(^{6}\right)$, et bien d'autres ensuite, les $p t$ coincïdent en général avec la phase croissante de la baie ou la précèdent un peu; selon certains auteurs il arriverait aussi que des pulsations accompagnent la phase décroissante, mais le fait semble douteux. Citons encore le Comité international: "les baies claires et isolées qui apparaissent pendant une période calme, dont le début est avec ou sans pulsations, brusque ou non, seront notées $b$. Si une baie se présente au moins sur une composante, avec des pulsations précédant ou suivant son début avec une faible différence de temps de l'ordre de 5 minutes $(*)$, on la signalera comme $b p$ ».

faites en dehors de l'URSS ne confirment guère cette opinion, ou plutôt conduisent à concilier, comme nous le verrons plus loin, la simultanéité des phénomènes individuels avec un contrôle local de leur amplitude.

(*) Pouvant atteindre 10 minutes (résolution prise à l'assemblée de Toronto, 1957). 


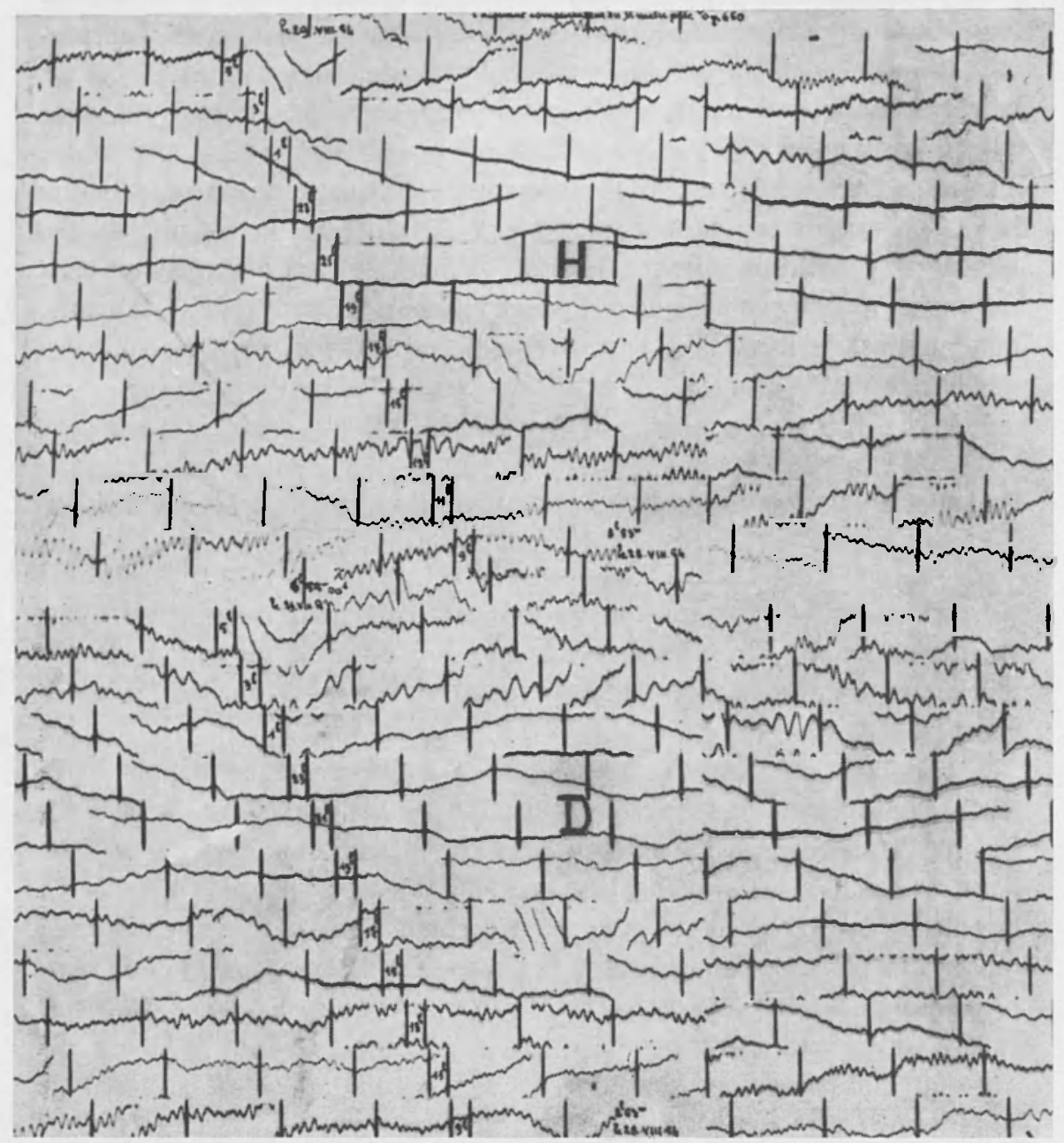

Fig. 1 - Aspect d'une journée peu agitée (Fragment d'enregistrement à Chambon-la-Forêt, 28 au 29 aout 1954; variomètres magnétiques horizontaux de Gibault, enregistreur rapide de La Cour). Il y a 5 minutes entre deux traits verticaux, environ deux heures entre deux lignes horizontales. 
Schématiquement, l'aspect d'une baie bien caractérisée ("claire») est celui de la fig. 2. La baie [2] est superposée à l'évolution diurne [1] qui peut être retrouvée par continuité sur les enregistrements lents. A la baie est superposée la pulsation [3] dont l'amplitude est exagérée sur le schéma; la séparation entre [2] et [3] peut se faire par continuite sur les enregistrements rapides. Le début brusque que les baies semblent souvent présenter est du en fait à la première oscillation de la pulsation.

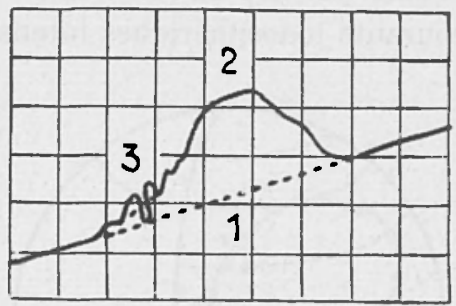

Fig. 2 - Schema de $b p$.

Plusieurs auteurs (notamment Terada $\left({ }^{7}\right)$; Hatakeyama $\left({ }^{5}\right)$; Kato et ses élèves $\left({ }^{8}\right)$; Scholte et Veldkamp $\left({ }^{9}\right)$ ont étudié la variation du champ au cours d'une $b p$. Vu l'amplitude très faible des pulsations et la vitesse très faible des déroulements sur la plupart des enregistrements actuels il faut accueillir avec prudence certaines assertions. D'ailleurs, même en admettant leur validité, elles peuvent dépendre de circonstances locales dues à la canalisation des courants telluriques par le sous-sol au voisinage de la station (cas des stations côtières par exemple), et la période même peut influer. En fait les descriptions sont passablement contradictoires en ce qui concerne la composante horizontale, et s'étendent rarement à la composante verticale. Les schémas de Veldkamp, établis dans deux cas seulement mais pour un grand nombre d'observatoires, semblent impliquer que la composante horizontale de la pulsation ([3] de la fig. 2) est polarisée, mais dans des directions très variables. Au contraire les reconstitutions faites par Kato et al. en 1953 montrent des sortes d'ellipses extrêmement irrégulières, très allongées dans le sens NS $\left(^{*}\right)$. Hatakeyama trouve franchement des ellipses et il affirme que la rotation dans le sens des aiguilles d'une montre prédomine le matin et le soir, tandis que la rotation inverse prédomine un peu après midi ou après minuit; la direction du grand axe

(*) C'est ce qu'on trouve aussi à la station Charcot. 
de l'ellipse serait celle du vecteur perturbation. D'après un autre article de Kato, Ossaka, Watanabe, Okuda et Tamao $\left({ }^{10}\right)$, la polarisation serait rectiligne dans la moitié des cas seulement, et en se bornant aux 2 ou 3 premières oscillations. Leur direction serait inclinée d'un angle variable sur la direction NS; les données suggèrent vaguement que les points où cette direction coupe les zones aurorales ne participeraient pas à la rotation terrestre.

Iues baies sont, comme on sait, un phénomène des latitudes moyennes, produit par des courants ionosphériques intenses mais très localisés

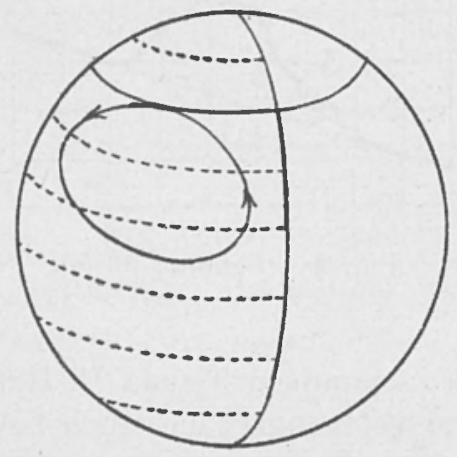

Fig. 3

dirigés d'Est en Ouest le long d'un segment nocturne de la zone aurorale, se fermant par une nappe de courants circulant dans les régions tempérées et allant même jusqu'aux régions tropicales (fig. 3). Dans la zone aurorale, on n'observe en général qu'une agitation désordonnée. En descendant vers l'équateur, l'aspect se simplifie, la baie se forme; puis elle s'atténue et disparaît; mais la pulsation conserve son amplitude. Si l'on passe de l'hémisphère nocturne à l'hémisphère diurne, la baie augmente et se complique de nouveau, présentant parfois les phénomènes appelés " vortex " ou " OSII ", puis disparaît encore; l'amplitude des pulsations conjointes varie, de facon mal connue, mais elles ne disparaissent pas en général. Ainsi les $p t$, si elles s'observent mieux de nuit, sont un phénomène mondial et beaucoup d'entre eux, observés seuls, ou peutêtre même la totalité, seraient en d'autres lieux accompagnés d'une baie.

D'une station à l'autre on peut reconnaitre que les baies sont à peu près simultanées tant que leur forme ne s'altère pas trop. La simultanété des $p t$ est beaucoup mieux définie. Angenheister trouve sur des milliers de kilomètres, de Java à Samoa par exemple, des écarts inférieurs aux 
erreurs possibles dans ses déterminations, erreurs qu'il estime à 2 secondes environ, mais qui devaient être au moins d'une dizaine de secondes. Kato et Ossaka (') trouvent 22 coïncidences à quelques secondes près entre des pt enregistrées à Onagawa pendant l'année 1950 sur un système barre-galvanomètre et à Tamanrasset sur un système Grenet-Castet. Le premier étant moins sensible il semble que les auteurs y aient considéré les $p t$ nocturnes et les aient retrouvés tant bien que mal à Tamanrasset, qui se trouvait en général éclairé $\left(^{*}\right)$.

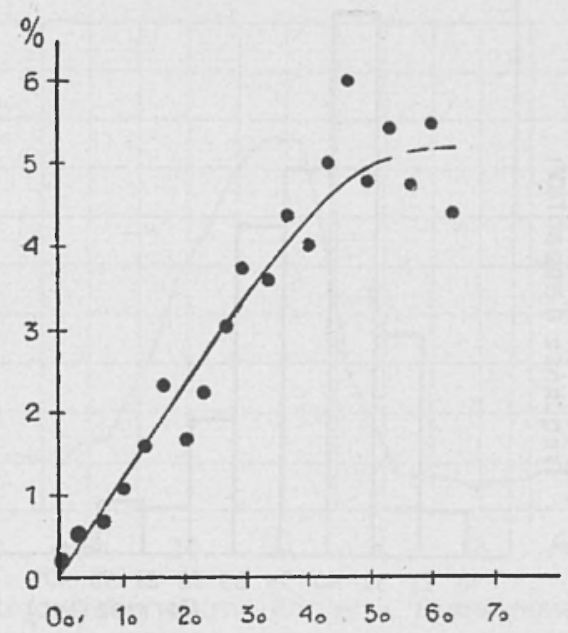

Fig. 4 - Fréquence d'apparition des $p t$ pour chaque valeur du caractère $K p$ (d'après Yanagihara).

Si elles ne correspondent pas toujours à des baies, les $p t$ sont néanmoins liées à des perturbations magnétiques de même genre, en général d'importance moyenne. Yanagihara $\left({ }^{12}\right)$ a étudié sur les enregistrements telluriques de Kakioka (Japon) la fréquence d'apparition des $p t$ dépassant un certain seuil d'amplitude en la comparant au caractère magnétique $K_{p}$ (fig. 4). La fréquence croît rapidement jusque vers $K_{p}=5_{o}$, mais semble décroître ensuite.

G. Angenheister à Apia, puis J. L. Bureau à Tamanrasset, trouvent que les $p t$ clairs (avec ou sans baie) commencent par une croissance de

${ }^{*}$ ) Les longitudes des deux stations diffèrent de $136^{\circ}$, les longitudes géomagnétiques de $73^{\circ}$ seulement. Tananrasset est dans la zone tropicale où les po nasquent moins les $p t$. 
$H$. Cette propriété est sans doute exacte aux basses latitudes. Dans nos régions les débuts des $p t$ sont trop rarement nets pour qu'on ait pu énoncer une conclusion générale.

La statistique des $p t$ ne peut être faite que sur des appareils sélectifs, ce qui est très gênant pour en tirer des conclusions générales, même en ce qui concerne les périodes. L'aspect nocturne des enregistrements dits rapides est dû essentiellement aux périodes de l'ordre de la minute, qui

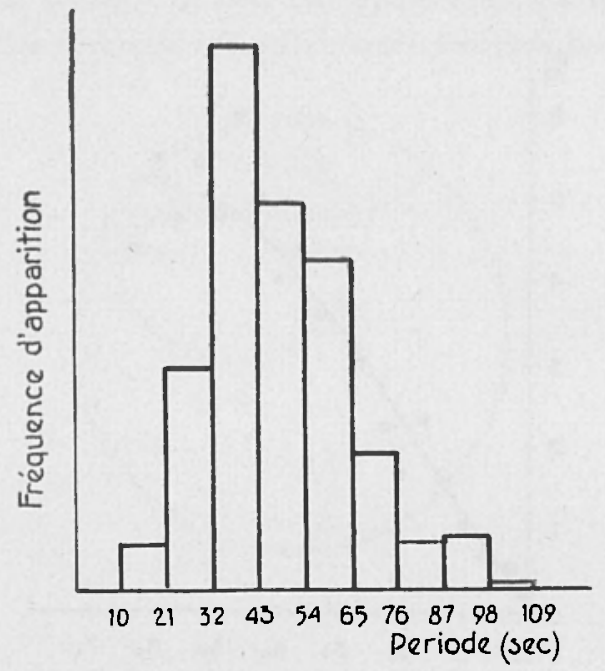

Fig. 5 - Fréquence d'apparition des $p t$ suivant leur période (d'après Kato et al.).

correspondent notamment aux $p t$ accompagnant les baies. Mais même sur ces enregistrements on trouve en général, superposées aux précédentes, des périodes de quelques secondes correspondant à des amplitudes très faibles. Si on en tient compte, comme naguère Kato et Ossaka $\left({ }^{13}\right)$, au Japon, ou comme Chernosky, Coon, et Maple $\left({ }^{14}\right)$ dans l'Arizona, la fréquence d'apparition apparaît d'autant plus grande que la période est plus courte. Si on considère seulement l'oscillation principale, comme l'ont fait Kato, Ossaka, Watanabe, Okuda, et Tamao $\left({ }^{10}\right)$ on obtient un maximum vers 40 sec (fig. 5); les domaines de périodes des $p c$ et des $p t$ ont donc une partie commune importante.

Van Bemmelen a montré que la période des $p t$ observés à Batavia sur des enregistrements lents, croissait de l'hiver à l'été. De même Holmberg trouve à Eskdalemuir que le centre de la bande de périodes est à 56 sec en hiver, à 71 sec aux équinoxes, à 78 en été. 
Comme la statistique des périodes, celle des amplitudes et celle des fréquences d'apparition sont viciées pas la sélectivité des appareils, malgré l'adoption de facteurs de réduction souvent assez incertains. La fréquence d'apparition, qui peut sembler a priori dépendre moins étroitement de ces facteurs, est liée à l'amplitude des plus faibles variations décelables. En gros, tout dépend de la position de la station par rapport aux tempêtes polaires qui sont, dans la zone aurorale, l'origine des $b p$, et par conséquent tout dépend du temps local bien que les tempêtes polaires

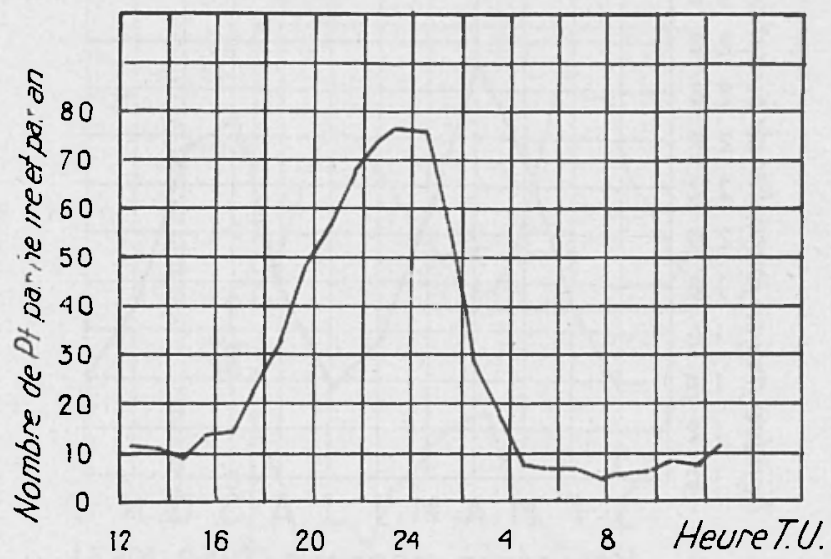

Fig. 6 - Répartition diurne des $p t$ à Tamanrasset de 1950 à 1957 (d'après Billaud et Bureau).

se succèdent fortuitement. Ainsi la fréquence d'apparition des $p t$ montre en fonction $d u$ temps local le grand maximum nocturne connu depuis longtemps pour les baies, avec un faible maximum secondaire de jour qui apparaît nettement dans les statistiques anciennes ou dans celle de Kato à Onagawa, moins nettement à Tamanrasset (fig. 6) ou à Kakioka et qui correspond peut-être à celui des baies négatives de la composante horizontale $\left(^{16}\right)$.

De même, la variation annuelle des $p t$ montre les maximums équinoxiaux de l'activité magnétique qui apparaissent aussi dans la variation annuelle des baies, mais cette propriété des $p t$, très nette à Tamanrasset (fig. 7) et à Kakioka est obscurcie à Wingst et à Onagawa par une tendance générale à un maximum d'hiver.

Comme la plupart des phénomènes géomagnétiques, y compris les baies, les $p t$ montrent la récurrence des 27 jours correspondant à la rotation $\mathrm{du}$ soleil. Cependant Yanagihara $\left({ }^{17}\right)$, conservant seulement les $p t$ 
telluriques d'amplitudes supérieure à un seuil assez élevé, ne trouve plus trace $d u$ phénomène, ce qui fait croire à une baisse rapide d'amplitude au cours des récurrences successives.

Pour les mêmes pulsations Yanagihara trouve que la fréquence d'apparition des $p t$ varie en sens inverse de l'activité solare (fig. 8) ce qui lierait les $p t$ aux régions $M$ de Bartels, c'est à dire à la cause hypothétique des tempêtes magnétiques en années de minimum des taches. On pour-

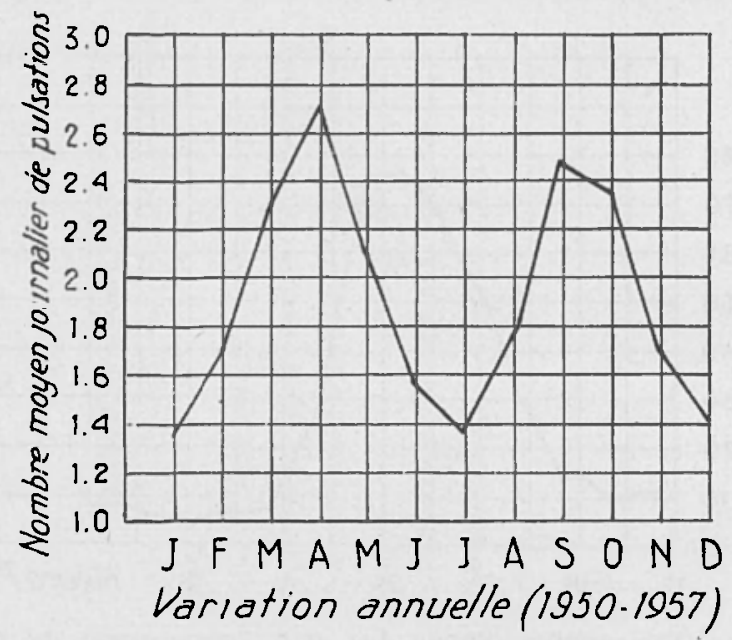

Fig. 7 - Variation annuelle des pt à Tamanrasset de 1950 à 1957 (d'après Billaud et Bureau).

rait penser tout simplement qu'elles se voient mieux en années de moindre activité. Cependant voici les statistiques de Tamanrasset, Agincourt et Meanook.

\begin{tabular}{|c|c|c|c|c|c|c|c|c|}
\hline & 1950 & 1951 & 1952 & 1953 & 1954 & 1955 & 1956 & 1957 \\
\hline Nombre de Wolf. & 84 & 69 & 31 & 14 & 4 & 42 & 142 & 178 \\
\hline Tamanrasset : & 819 & 945 & 646 & 656 & 941 & 902 & 226 & 201 \\
\hline Agincourt . & & 94 & 131 & 128 & 168 & 521 & & \\
\hline Meanook. . & & 29 & 101 & 194 & 165 & 489 & & \\
\hline
\end{tabular}

Bien que les définitions utilisées soient peu comparables et qu'une réduction de sensibilité pendant les dernières années de Tamanrasset ne soit pas exclue, le moins qu'on puisse dire est que la loi de Yanagihara n'est pas ici vérifiée. Elle n'est peut-être valable que pour les $p t$ de grande amplitude. 
Pour améliorer l'étude statistique des $p t$ il serait utile de trier des cas purs. Une tentative en ce sens a été faite par Billaud dans le dépouillement des pulsations enregistrées sur le variomètre Grenet-Castet à Tamanrasset. Son type $P u$ débute par un accroissement exponentiel de $d H / d t$ durant environ 40 sec.; $d H / d t$ décroît alors brusquement, effectue une oscillation de grande amplitude, dont la pseudo-période serait de l'ordre de 30 secondes, ce qui est court pour des $p t$; puis l'oscillation s'amortit en devenant irrégulière. Dans les cas les plus nets le phénomène dure

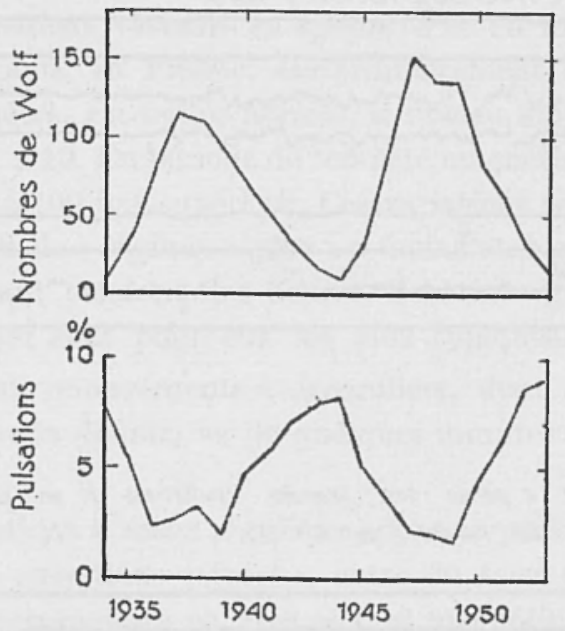

Fig. 8 - Activité solaire et nombre de pulsations à Kakioka (d'après Yanagihara).

environ 5 minutes. Grenet $\left(^{18}\right)$ retrouve à Chambon-la-Forêt les $P u$ de Tamanrasset, mais avec des changements capricieux d'amplitude et de pseudo-période. Bureau a d'ailleurs été conduit à des subdivisions du type (fig. 9).

Phénomènes diURnes des Latitudes moyennes: $p c$.

Revenons à notre enregistrement magnétique rapide, par jour un peu agité (fig. 1). Ce qu'il montre pendant le jour, surtout le matin, ce sont les $p c$, plus ou moins régulières, avec en général des périodes de 20 à 30 sec. Les principaux résultats concernant les $p c$ n'ont d'ailleurs pas été obtenus par voie magnétique. Ils sont dûs aux enregistrements telluriques de la Compagnie Générale de Géophysique, faits depuis 1941, 

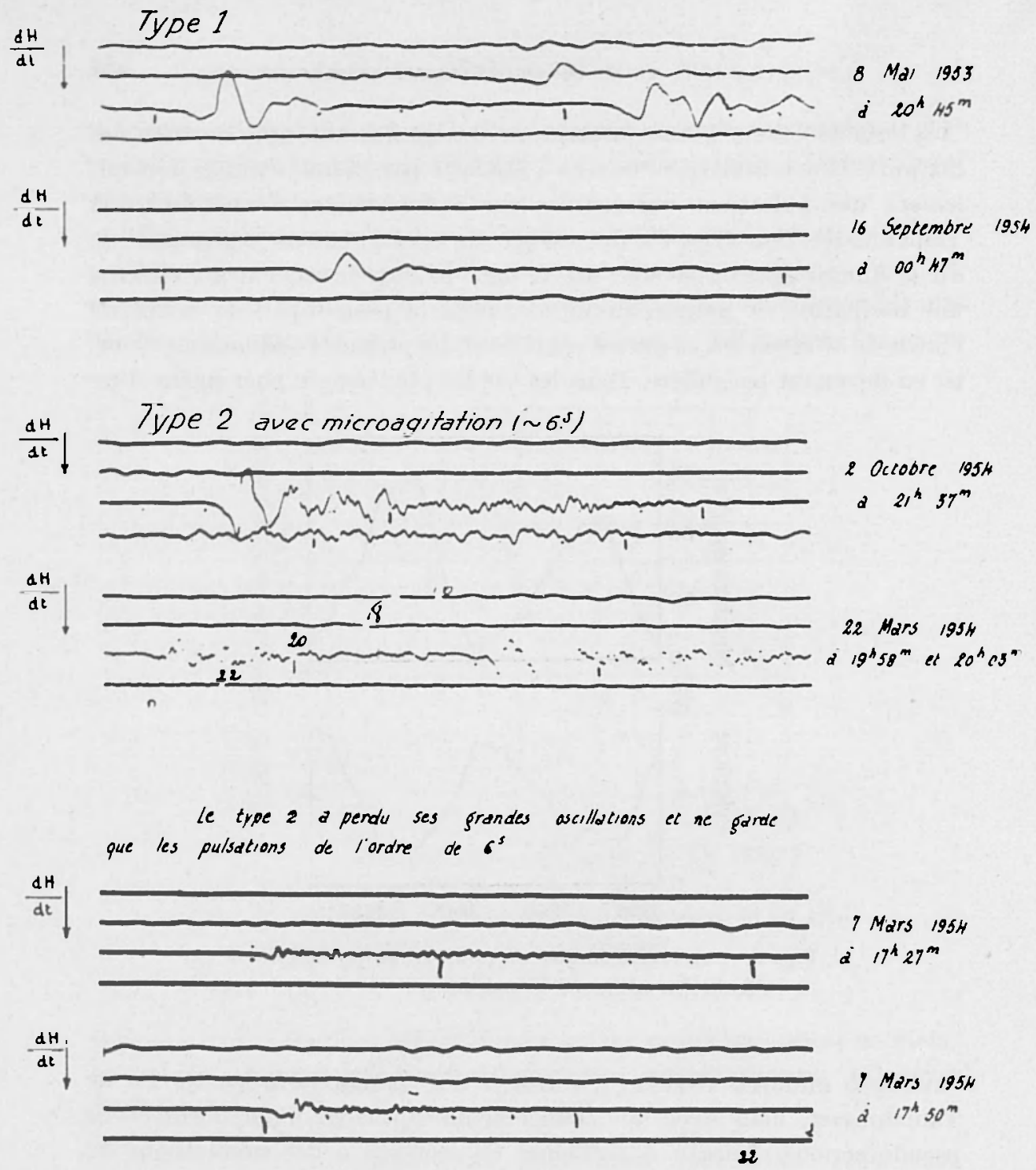

Le type 1 est preceide par des oscillusions floves

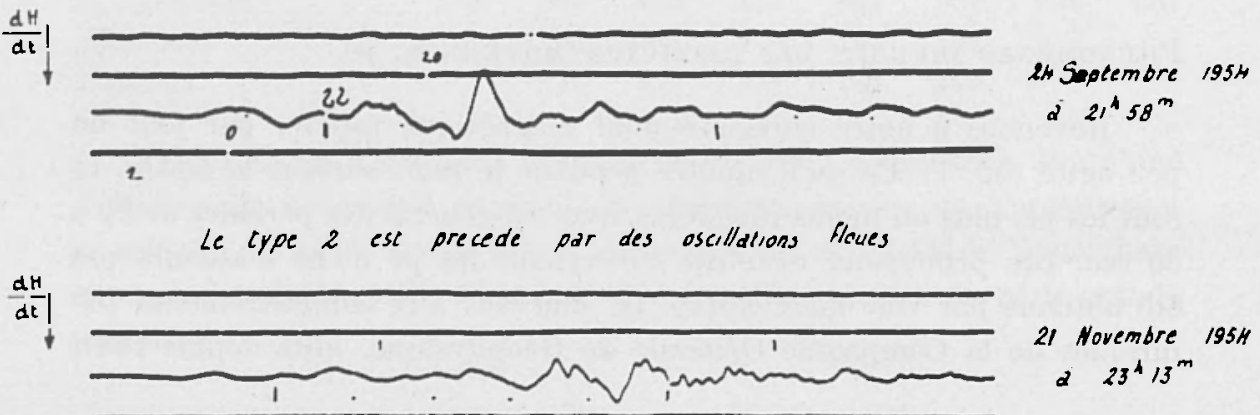

Fig. 9 - Types de pt (d'après Bureau). 
dans le monde entier, pour un but de prospection, et étudiés prineipalement par G. Kunetz $\left(^{19}\right.$ ) (à qui nous empruntons la plupart des détails qui suivent). La vitesse de déroulement $(2 \mathrm{~cm} /$ minute) et la constante de temps des galvanomètres (de l'ordre de la seconde) permettent aux prospecteurs de bien observer les phénomènes de période comprise entre quelques secondes et quelques minutes. Malheureusement l'heure est rarement connue avec précision, et leurs enregistrements sont presque tous faits de jour.

A la surface du sol le champ électrique est un vecteur, pratiquement horizontal, extrêmement variable en intensité et en direction. L'amplitude de ses variations, en France, est ordinairement de l'ordre de $\mathbf{0 , 5}$ millivolt par kilomètre. En temps normal, le niveau moyen ne sort guère d'une gamme de 1 à 10 . En période de tempête magnétique, 2 à 3 fois par an, il peut être 50 à 100 fois supérieur. Ces variations se présentent comme la superposition de "régimes " plus ou moins stables, dont l'un peut devenir prépondérant pendant des heures. Schlumberger et Kunetz ${ }^{\left({ }^{20}\right)}$ énumèrent ceux qui sont pour eux les plus typiques. Ils distinguent:

- Les "grands mouvements ", irréguliers, dont la période, pour autant qu'on puisse la définir, va de quelques minutes à quelques dizaines de minutes.

- Les "pulsations ", assez régulières, dont la période est voisine de la minute et les "pulsations serrées", entre 30 secondes et 1 minute. L'ensemble doit correspondre en gros aux $p t$ magnétiques.

— Les "oscillations normales ", qui correspondent aux pc magnétiques ordinaires, s'observent assez souvent à l'état pur et se distinguent par leur très grande régularité. Leur période, stable à quelques secondes près (parfois à la seconde près pendant plusieurs heures consécutives) est comprise entre 12 et 30 secondes environ. La forme de chaque onde est symétrique: l'intensité, assez constante en moyenne, fluctue généralement en formant des successions de groupes d'ondes; c'est l'aspect caractéristique des phénomènes à spectre étroit.

- Les " oscillations serrées ", plus rares, descendent jusqu'à 6 secondes, et semblent alors disparaître, tout au moins dans les observations de la C.G.G. Cependant, en disposant d'appareils convenables on peut mettre en évidence des périodes plus courtes comme celles des pulsations, montrant toujours la structure en groupes, observées par Mme Troïtzkaïa entre 2 et 0,3 sec et par Selzer jusqu'à 0,1 sec.

Sans conserver la terminologie de Schlumberger et Kunetz on retiendra de leur description que les pulsations diurnes correspondent 
à une superposition de bandes de fréquence privilégiées. La mieux définie, celle des $p c$, n'est pas, explique Kunetz, limitée à ses deux extrémités de la même façon: "Si l'on trouve très souvent des pulsations de période supérieure à 30 ou 35 secondes, elles deviennent très vite beaucoup moins régulières. On n'a jamais observé un train de 5 oscillations régulières d'une période supérieure à 50 sec. A l'autre extrémité de la bande il existe des oscillations extrêmement régulières d'une période de 7 ou 8 secondes mais on en rencontre 50 ou 100 fois moins souvent que dans la bande habituelle ".

Comme dans le cas des $p t$ on ne peut évidemment séparer l'étude des périodes de celle des intensités: si l'on observe à un moment des oscillations presque pures c'est que leur intensité est suffisante pour masquer les autres phénomènes. Kunetz constate d'ailleurs que pendant des perturbations même moyennes, le phénomène oscillatoire est toujours présent, le plus souvent relativement pur. Il est même fréquent que, pendant des tempêtes magnéto-telluriques violentes, ce phénomène reste prédominant: en apparence l'enregistrement tellurique correspondant ne diffère en rien d'une journée "oscillatoire " normale mais la sensibilité a dú être réduite dans un rapport de 20 ou 50 . Inversement, au cours des journées très calmes on trouve rarement le phénomène oscillatoire: s'il existe, il doit être noyé dans un fond de variations diverses.

L'étude de la largeur du spectre a été abordée surtout par Holmberg $\left({ }^{3}\right)$, sur des enregistrements magnétiques; il énonce la loi suivante: le rapport de la largeur de bande à la fréquence médiane varie peu avec la période sur l'ensemble des périodes de 15 à 100 secondes (Holmberg englobe les $p t$ dans la statistique). Il est de l'ordre de 0,20 à 0,25 .

La relation statistique entre la période et l'intensité moyenne des pulsations a été étudiée par Holmberg et beaucoup plus longuement par Angenheister $\left({ }^{21}\right)$. Ils ont trouvé tous deux une croissance rapide de l'intensité moyenne avec la période, qu'ils ont d'ailleurs étendue à des périodes élevées pour lesquelles il ne peut s'agir de véritables $p c$ régulières. La question devrait être reprise en distinguant soigneusement les $p t$. Holmberg a également determiné la fréquence d'apparition des diverses amplitudes au cours de $p c$ ayant duré plus d'une heure. Il trouve des distributions de Rayleigh $p(x)=x / S$. $\exp \left(-x^{2} / 2 S\right)$.

Holmberg trouve d'autre part qu'aux heures où apparaissent beaucoup de pulsations leur intensité moyenne est plus grande ce qui s'interprète bien par un relèvement général d'intensité amenant des pulsations faibles au-dessus du seuil de perceptibilité. 


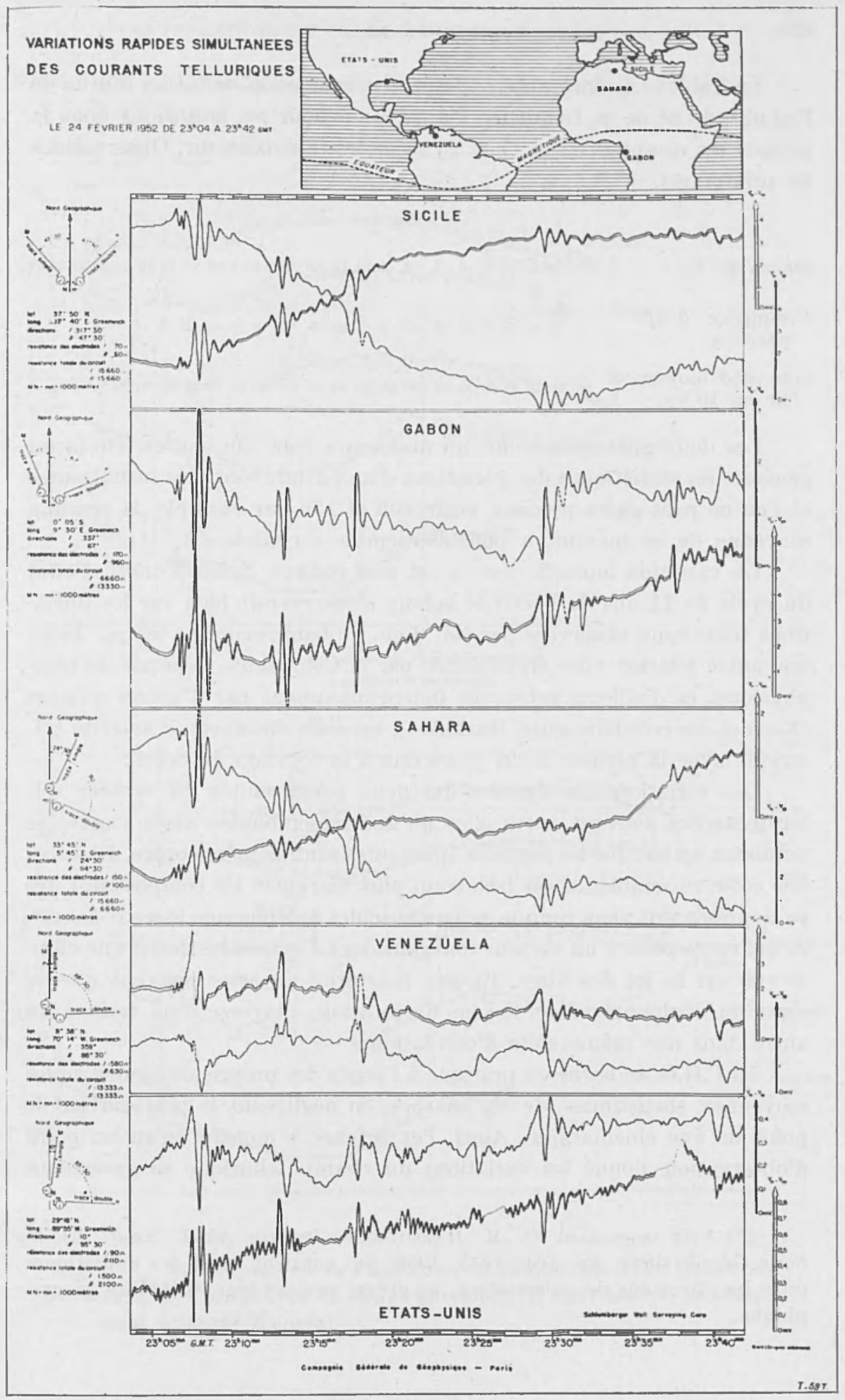

Fig. 10 - Corrélations telluriques à 'grande distance le 24 février 1952 (d'après Kunetz). 
Le Tableau I, emprunté à Holmberg, montre la variation diurne de l'amplitude et de la fréquence d'apparition pour les pulsations dont la période est comprise entre 17 et 26 secondes (Eskdalemuir, Observations de printemps).

\section{Tableau I}

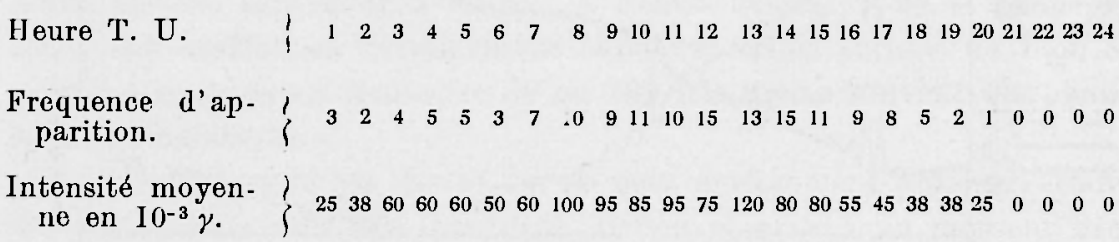

Les deux phénomènes ont un maximum ver's 13 h locales. De façon générale les statistiques des variations d'amplitude sont très insuffisantes et l'on ne peut guère préciser, entre $10 \mathrm{~h}$ et $15 \mathrm{~h}$ par exemple, la position moyenne de ce maximum pour l'ensemble du Globe (*).

La variation annuelle des $p c$ est mal connue. Selon Kunetz, l'effet du cycle de 11 ans de l'activité solaire s'observerait bien sur les pulsations telluriques observées par lui, done probablement sur les pc. Enfin une autre relation mise en évidence par la Compagnie Générale de Géophysique, et d'ailleurs retrouvée indépendamment par d'autres auteurs (Kato et ses collaborateurs, Burkhart), est celle du niveau d'activité tellurique avec la période de 27 jours due à la rotation du Soleil.

Les variations simultanées des deux composantes du vecteur tellurique n'ont souvent entre elles qu'une ressemblance assez vague, se réduisant au fait que les périodes apparentes sont de même ordre. D'autres fois cette ressemblance est beaucoup plus marquée: les composantes des $p c$ se présentent alors comme deux sinusoïdes à déphasage assez constant, ce qui correspond à un vecteur tournant dont l'extrémité décrit une ellipse suivant la loi des aires. Encore constate-t-on assez souvent que le signe du déphasage, donc le sens de rotation, s'inverse d'un tram à un autre dans une même suite d'oscillations.

La C.G.G. se borne en pratique à l'étude des propriétés géométriques moyennes, statistiques, de ces courbes, en négligeant le plus souvent le point de vue cinématique. Ainsi, l'expérience a montré qu'en un point d'observation donne les variations du champ tellurique se groupaient

(*) Voir cependant O. M. BARsoukov, Izvestia Akad. Naut. SSSR, Serie Géoplyysique, pp. 1040-1043, 1958, qui contient aussi des statistiques pour les directions de polarisation, montrant surtout leur variabilité géographique. 


\section{Niveau moyen de l'agitation tellurique}

( corrdlationa en G.M.T. )

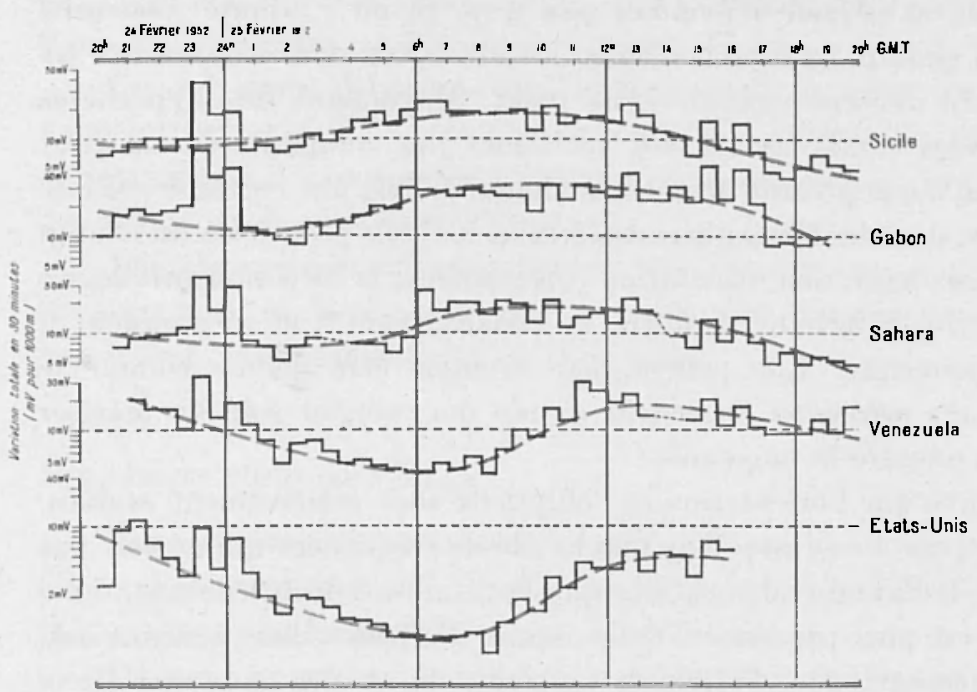

Nivedu moyen de l'agitation tellurique (corraldatlong en heure localo)

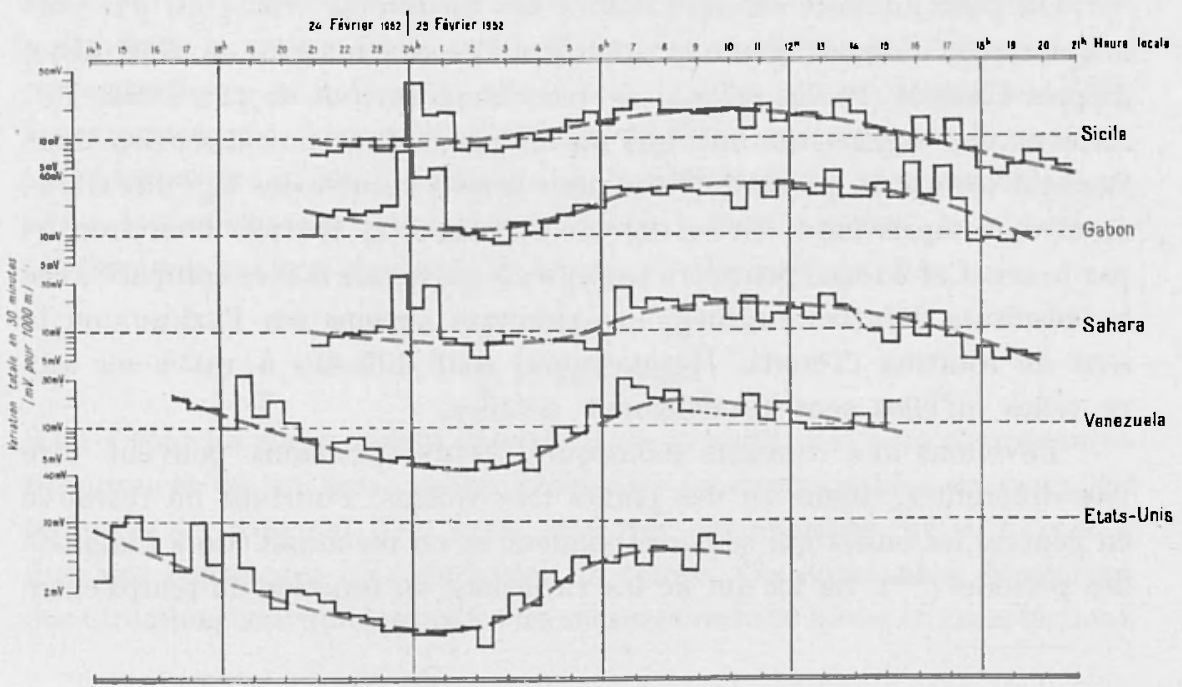

Fig. 11 - Niveau moyen de l'agitation tellurique, les 24 et 25 février 1952. 1) Corrélations en temps universel; 2) Corrélations en temps local (d'après Kunetz). 
souvent de préférence autour d'une direction moyenne déterminée et que le degré $d u$ resserrement autour de cette direction était relativement stable tant que la période n'évoluait pas trop. Si on y ajoute l'intensité moyenne du champ, on a trois éléments statistiques pour caractériser les variations $d u$ vecteur champ en ce point. Moyennant des lyypothèses ussez générales sur la nature des variations des composantes, on peut définir d'une façon précise, attachée à chaque point, une certame « ellipse intrinsèque ", dont les éléments caractérisent les trois propriétés du champ indiquées plus haut; son orientation correspond à la direction privilégiée du champ, son ellipticité au degré de canalisation $\left(^{*}\right)$ et sa grandeur à l'intensité moyenne. Elle pourra, par exemple être définie comme le lieu moyen des extrémités du vecteur champ (en prenant soin de préciser la façon de prendre la moyenne).

On trouve que l'orientation et l'ellipticité sont relativement stables, surtout tant que l'on reste dans une bande de fréquences qui ne soit pas trop large, et d'autant plus stables que la canalisation du courant, done l'ellipticité est plus prononcée. Celle-ci peut d'ailleurs, dans certains cas, être extrêmement forte, l'intensité moyenne du champ dans une direction particulière étant 10 ou 20 fois supérieure à celle dans la direction perpendiculaire.

On pourrait bien entendu définir des propriétés analogues par voie magnétique, mais les résultats actuels sont assez maigres. A Gottingen, d'après Untiedt ${ }^{(22}$ ), les $p c$, qui se recontrent surtout de $12 \mathrm{~h}$ à $15 \mathrm{~h} \mathrm{TU}$, forment des oscillations linéaires ou allongées dans une direction dont l'azimut (compté à partir du Nord dans le sens inverse des aiguilles d'une montre) compris entre $10^{\circ}$ et $90^{\circ}$ décroît dans la journée d'environ $3^{\circ}$ par heure. Cet énoncé peut-être trop précis gagnerait à être comparé avec les résultats d'autres stations. Les résultats anciens sur l'azimut ou le sens de rotation (Terada, Hatakeyama) sont difficiles à rattacher aux $p c$ telles qu'elles sont actuellement définies.

Revenons aux courants telluriques. Leurs variations peuvent être très différentes, même en des points très voisins. Pourtant on retrouve en général les ondes qui se correspondent et on reconnaît alors l'identité des périodes $\left({ }^{* *}\right)$. La loi qui lie les variations en fonction du temps entre

$\left(^{*}\right)$ Ce terme employé par les prospecteurs n'implique pas que la "canalisation " soit due au sol: l'ampleur des fluctuations d'orientation du champ est évidemment licé à l'origine de la pulsation.

$\left({ }^{* *}\right)$ Signalons cependant qu'au cours d'une comparaison entre les pulsations magnétiques observées à Chambon-la-Forêt (Loiret) et à l'Observatoire 
les deux points $A$ et $B$ est alors de forme simple: si l'on considère le lieu décrit par l'extrémité du vecteur ehamp en $B$, il se déduit du lieu analogue en $A$ par une rotation suivie d'une double dilatation dans deux directions rectangulaires, stables dans le temps.

Ces rotations, de même que les dilatations, peuvent être extrêmement importantes: dans des régions à géologie tourmentée on a observé des variations d'intensité de 1 à 100 et des rotations de $90^{\circ}$ et plus sur des distances de quelques kilomètres.

Fort heureusement pour l'étude des corrélations, il n'en est pas toujours ainsi et, le plus souvent, les projections sur des lignes parallèles présentent une ressemblance assez grande.

\section{Relations Fondamentales.}

Pour en revenir à la relation entre champ en deux points, nous avons d'abord donné celle qui he les diagrammes polaires, car elle est indépendante de la manière de mesurer le champ.

On peut exprimer cette relation d'une façon également très simple sur les composantes, à condition d'admettre ce qui suit: Chaque fois que le champ en un point prend une certame direction, le champ simultané en un autre point reprend lui aussi une même direction, d'ailleurs différente de la première (autrement dit en deux points quelconques il y a correspondance bi-univoque entre les directions des champs); de plus, les intensités du champ suivant deux de ces directions homologues restent proportionnelles. On en déduit immédiatement que les relations entre les composantes du champ suivant deux couples de directions absolument quelconques sont linéaires, c'est-à-dire que l'on a:

$$
\begin{aligned}
& X=a x+b y \\
& Y=c x+d y
\end{aligned}
$$

$X$ et $Y$ sont les composantes du champ en un point, $x$ et $y$ les composantes simultanées en un autre point, toutes les quatre variables au cours $d u$ temps, mais toujours liées entre elles par ces relations, où $a, b, c$ et $d$ sont des constantes indépendantes du temps. Ces constantes dépendent des directions suivant lesquelles les mesures ont été faites et caractérisent

de Haute Provence, distants de $500 \mathrm{~km}$, les périodes à (hambon se sont montrés supérieures dans un très petit nombre de cas; les ondes correspondantes étaient probablement masquées en Haute Provence par les périodes plus courtes $\left({ }^{23}\right)$. 
mal la liaison entre les deux points; mais on peut en déduire des invariants, par exemple ceux qui ont été indiqués à propos du diagramme polaire. En particulier, le produit des deux dilatations dont on a parlé plus haut, qui est en somme le rapport des surfaces de deux boucles homologues quelconques des deux diagrammes, soit $a d-b c$, est la paramètre le plus utilisé, sous le nom d'aire, pour différencier les terrains en prospection tellurique.

Si l'aire relative au point $A$ est grande en un point $B$ c'est que l'épaisseur des sédiments est plus faible en $B$ qu'en $A$, parce que le fond cristallin qui est électriquement résistant y canalise les lignes de courant au voisinage de la surface.

Malheureusement de nombreux facteurs, notamment les courants vagabonds industriels, perturbent les phénomènes. Ils introduisent une dispersion dans la détermination de l'aire. Celle-ci est définie au mieux à $10 \%$ près dans un rayon de $50 \mathrm{~km}$.

Une étude quantitative de la corrélation entre les vecteurs champ en deux points, corrélation beaucoup plus complexe que la corrélation scalaire ordinaire, a été présentée par Bessière comme Diplôme d'Etudes supérieures à la Faculté des Sciences de Paris, mais non publiée. Il a trouvé des coefficients de corrélation encore très élevés, supérieurs à 0,80 , jusqu'à des distance de 100 à $200 \mathrm{~km}$, puis décroissant jusqu'aux environs de 0,50 quand on atteint des distances d'un millier de kilomètres. A partir de ces distances, non seulement la qualité des corrélations diminue rapidement, mais encore cette qualité devient très variable dans le temps: alor's qu'à certains moments on trouve encore des corrélations très nettes, à d'autres, d'autant plus nombreux que la distance augmente, toute corrélation parait cesser. On constate également que les corrélations sont perceptibles sur des distances plus grandes dans le sens Nord-Sud que dans le sens Est-Ouest.

Dans le sens NS on a trouvé par exemple des similitudes frappantes entre des trains d'oscillations observés simultanément en Europe et à Madagascar, la rotation du vecteur champ se faisant généralement, mais non pas toujours $\left({ }^{24}\right)$ en sens inverse dans les deux hémisphères. On trouve également d'excellentes corrélations entre les niveaux moyens d'activité. $\mathrm{Au}$ contraire, les corrélations dans le sens EW posent immédiatement une question que nous avons déjà débattue: les phénomènes doivent-ils être rapportés au temps universel ou au temps local? La réponse est que les deux interviennent. L'influence de l'heure locale est évidente: les $p c$ ne sont guère visibles que pendant le jour, les $p t$ que pendant la nuit, que celles-ci soient plus faibles le jour ou masquées par les pc. En revan- 
che, les corrélations de détail entre oscillations, chaque fois qu'on arrive à mettre ces corrélations en évidence, correspondent à une même heure du temps universel, même s'il y a entre les points d'observation une différence de longitude équivalant à plusieurs heures (fig. 10).

Des identifications analogues aux précédentes ont été obtenues sur des enregistremants magnétiques par Kato et Okuda $\left({ }^{25}\right)$ entre le Japon et Ceylan.

La dualité qui apparaît en ce qui concerne les pulsations individuelles s'observe également sur l'agitation moyenne. Sur cinq enregistrements de février $1955\left(^{*}\right)$, Kunetz a évalué cette agitation moyenne $1 / 2$ heure par $1 / 2$ heure (c'est la "variation totale " $V$, somme des valeurs absolues des différences entre maxima et minima, qui a servi de mesure pour cette agitation). On trouve (fig. 11) que:

1. Quand on compare les courbes obtenues aux cinq stations, on constate une excellente corrélation en temps universel des variations brutales de cette activité en des points entre lesquels il existe jusqu'à des différences de sept heures d'heure locale.

2. On constate également une évolution lente du niveau d'activité, parallèle aux 5 stations, quand on aligne les courbes obtenues en heure locale.

Une manière d'expliquer ce double aspect du phénomène observé consiste par exemple à supposer que:

- d'une part, l'action directe d'un rayonnement corpusculaire provenant du Soleil, simultanément sur l'ensemble du Globe, produirait les corrélations, en temps universel, du détail des pulsations ou des fluctuations brutales de l'agitation.

- d'autre part, l'action du rayonnement électromagnétique du Soleil, en rapport avec l'heure locale, modifierait le niveau d'ensemble en agissant sur la conductivité de l'ionosphère.

\section{AUTRES PHÉNOMÈnes DES Latitudes MOYeNNES.}

Les baies d'une part, l'agitation magnétique modérée d'autre part, ne sont pas les seuls phénomènes magnétiques macroscopiques accompagnés de pulsations. Mais les descriptions se compliquent dès qu'il s'agit de véritables tempêtes magnétiques.

(*) A l'occasion d'une éclipse totale de Soleil. Disons en passant que l'effet d'une telle éclipse sur les pulsations, effet plusieurs fois recherché, n'a jamais pu être nettement mis en évidence. 
Les crochets magnétiques qui correspondent à l'effet ionosphérique des éruptions chromosphériques et qui peuvent être considérés comme les précurseurs de certaines tempêtes magnétiques, ne sont pas accompagnés de pulsations particulières. Par contre, si la tempête débute brusquement (ce qu'on note internationalement par ssc) ou si elle présente une recrudescence brusque d'agitation, on observe à ce moment sur les dispositifs à induction une discontinuité de pente (presque toujours
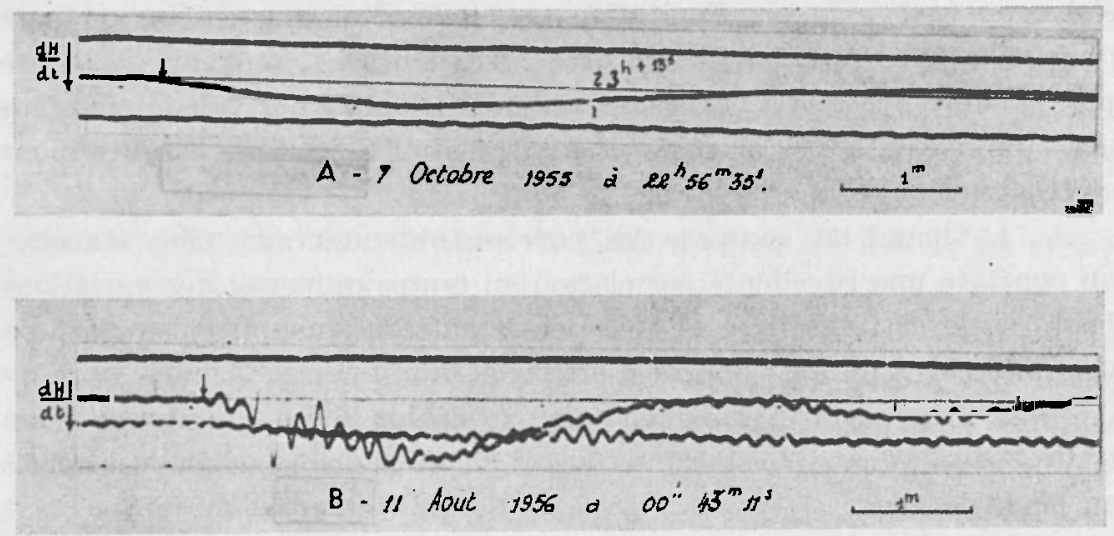

Fig. 12 - Débuts brusques de tempêtes magnétiques à Tamanrasset sur les variomètres électromagnétiques de Grenet (d'après Bureau).

vers les valeurs positives pour $d H / d t$ ) suivie d'une ou plusieurs oscillations amorties à période de plusieurs minutes (Fig. $12 \mathrm{~A}$ ). C'est ainsi du moins que le phénomène se présente à Tamanrasset $\left({ }^{28}\right)$. Dans 12 cas sur 93, des pulsations courtes sont présentes à son début (fig. $12 \mathrm{~B}$ ). Elles sont parfois très régulières. A Onagawa, Kato et Saito $\left({ }^{27}\right)$ ont étudié ces cas de " $p t$ bien régullèrement amorties" ("fine damped "). Elles seraient assez semblables somme toute au type $P u$ décrit plus haut, mais avec des périodes plus courtes. 33 cas ont été observés par Kato et Saito en deux ans, dont 5 accompagnant des ssc, les autres des recredescences plus ou moins nettes. La pseudo-période allait de 13 à 27 secondes seulement avec un maximum prononcé vers 19 (de grandes périodes sous-jacentes se voient aussi sur les reproductions). Le rapport d'amortissement était voisin de 0,85 (le temps de relaxation de 100 à $120 \mathrm{sec}$ ). Selon Kato et-Saito, ces pulsations semblent rares en hiver, mais ce qui les distingue surtout des $p t$, outre leur période plus courte, c'est qu'elles sont extrêmement faibles la nuit, leur fréquence d'apparition présentant un maxi- 
mum prononcé vers 9h locales (c'est le contraire pour l'amplitude du début brusque lui-même, qui est minimum vers 8 heures, maximum vers 23h, comme l'on montré 'il y a longtemps de Moidrey à Zi-Ka-Wei et Maurain à Paris).

En dehors du début brusque et des recrudescences, la phase principale de la tempête est accompagnée de variations rapides, amples et persistantes, en général très irrégulières mais au milieu desquelles apparaissent parfois des $p c$ à courte période. En règle assez générale, lorsque l'agitation augmente, les pulsations de période donnée semblent perdre leur régularité tandis qu'apparaissent des pulsations régulières de période plus courte.

\section{Phénomènes des RÉGions aurorales.}

Si on laisse de côté l'agitation irrégulière toujours présente dans la zone aurorale, le phénomène pulsatoire le plus caractéristique de ces régions est un phénomène rare: au cours d'une tempête magnétique surgit une oscillation ample et régulière qui peut durer d'une dizaine des minutes à un petit nombre d'heures; c'est ce qu'on appelle une " pulsation géante " $(p g)$. L'adjectif est dû à Rolf (1931) mais le phénomène a été observé par Birkeland dès 1900. Dans un petit nombre de cas, Harang $\left({ }^{28}\right)$ a pu montrer que l'extrémité du vecteur pulsant décrivait une ellipse dont le grand axe, à Tromsö, semble toujours voisin de la direction EW. L'amplitude ne reste d'ailleurs pas constante. L'enveloppe des oscillations présente souvent une forme en fuseau, simple ou répétée, constituant un groupe d'ondes à spectre étroit.

La période d'une pulsation géante est du même ordre que celle des $p t$, de 20 à 300 sec pour fixer les idées; pour une inême pulsation la période semble être la même en tout lieu.

Les amplitudes des $p g$ ne sont supérieures à quelques gammas qu'un très petit nombre de fois par an, en Scandinavie ou en Islande. Elles sont plus fortes en période de grande activité solaire. Elles diminuent rapidement dès qu'on s'éloigne de la zone aurorale. En Suède, la pulsation du 12 Septembre 1930, étudiée par Rolf, tombait de $34 \gamma$ à Abisko à moins de $1 \gamma$ à Lovö sur $1000 \mathrm{~km}$. A Godhavn (Groenland), quelques degrés au nord de la zone aurorale, aucune pulsation géante n'a été observée en 11 ans sur les magnétomètres La Cour du type rapide. Aucune non plus à Resolute Bay, dans une position analogue, pour une durée d'ailleurs plus brève. 
484

J. COULOMB

Obsevratoinews: de Thamenon -

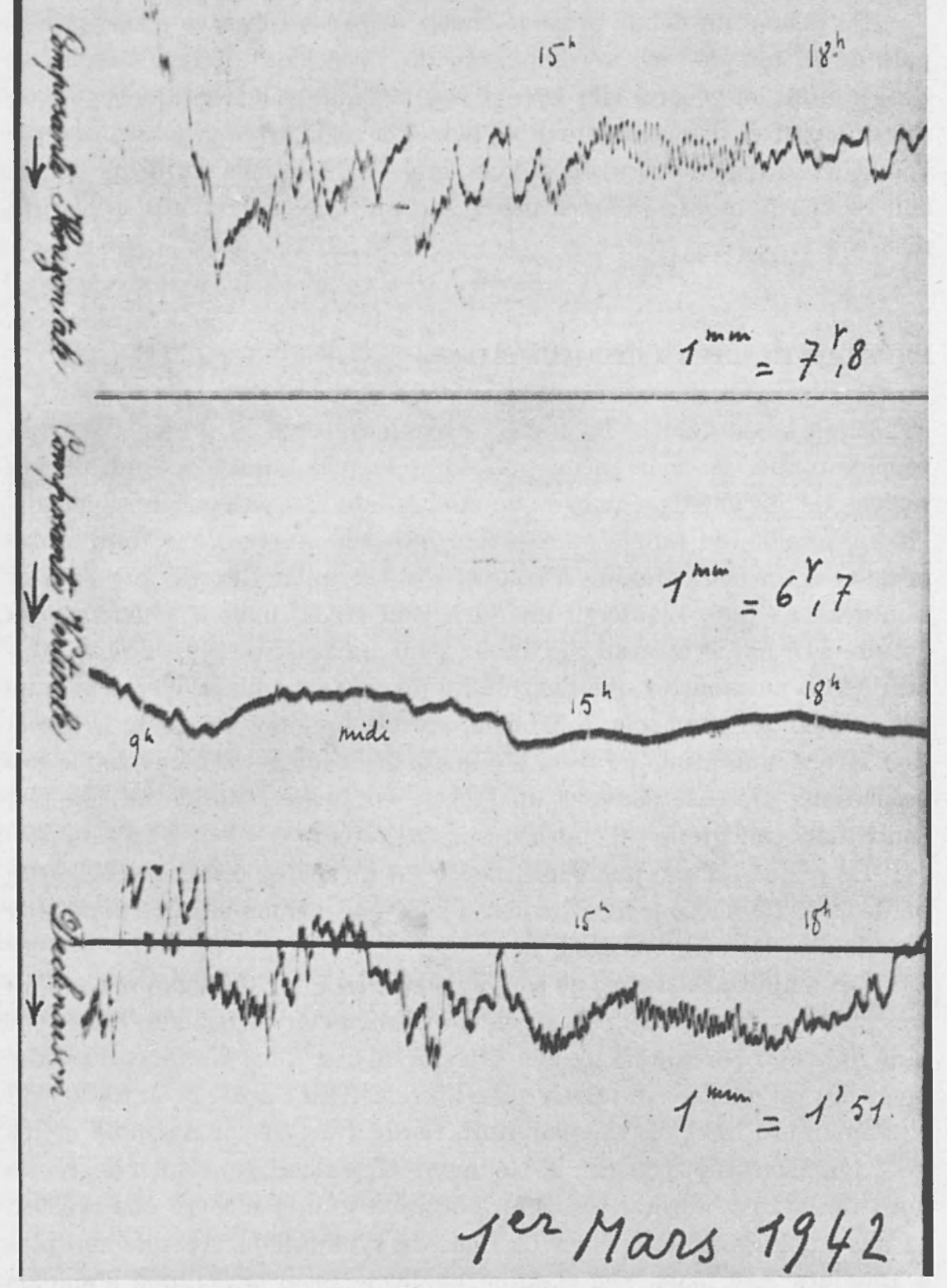

Fig. 13 - La pulsation géante du 1er Mars 1952 observée à Chambon-laForêt (variomètres Mascart). 
Les $p g$ peuvent cependant avoir une période et une amplitude exceptionnellement grandes; elles sont alors perceptibles à des latitudes plus basses. La pulsation du 1er Mars 1942 détient probablement le record des périodes avec 4 minutes et demie environ. Elle a été enregistré jusqu'à Tamanrasset sur des variomètres ordinaires. A Chambon-la-Forêt et à Nantes (fig. 13) où elle présentait grossièrement la forme en fuseau, l'amplitude atteignait $55 \gamma$ sur la composante horizontale.

Selon Sucksdorff ${ }^{\left({ }^{30}\right)}$ la fréquence des $p g$ varierait en sens inverse de l'activité solaire.

Les pulsations géantes ne sont pas les seules pulsations régulières des zone aurorales. Les pulsations observées par Chree dans l'Antarctique avaient des périodes en général plus courtes (descendant jusqu'à 7 secondes) et des amplitudes atteignant une fois seulement $8 \gamma$; elles n'ont guère de commun avec les pulsations géantes que leur régularité.

Harang et Sucksdorff ont décrit des types de pulsations courtes, non observées sur la composante verticale. Leur période est de l'ordre de la seconde. Elles se présentent assez différemment dans leurs deux stations: leur fréquence a un maximum pointu vers 10h à Tromsö, un maximum variant entre $10 \mathrm{~h}$ et $16 \mathrm{~h}$ à Sodankyla, où leur nombre est beaucoup plus grand. Ces observations fragmentaires font penser que la gamme de périodes des pulsations régulières de la zone aurorale peut s'étendre beaucoup plus bas.

On peut enfin se demander si les pulsations des régions aurorales boréale et australe sont simultanées. La question est importante pour la théorie mais elle n'a guère été abordée que dans un article assez décevant d'Ahmed et Scott.

\section{$R \mathcal{E S U M E}$}

L'article résume l'ensemble de nos connaissances expérimentales sur les pulsations magnétiques et telluriques.

\section{$A B S T R A C T$}

Review of observational knowledge on pulsations of magnetic field and tellurio currents.

\section{RIASSUNTO}

L'Autore riassume nell'articolo l'insieme delle conoscenze sperimentali sulle pulsazioni magnetiche e telluriche. 


\section{BIBLIOGRAPHIE}

(1) R. P. Romana, Provisional Atlas of Rapid Variations, Association Internationale de Géomagnétisme et d' $\Lambda$ éronomie, 1958.

$\left(^{2}\right)$ E. R. R. Holmberg, Monthly Not. Roy. Astr. Soc. Geophys., Suppl. 6, 467-481, (1953).

(3) V. A. Troitzkaia, Comptes-Rendus de l'Académie des Sciences de l'URSS (Doklady), XCIII, 261-264, (1953).

(4) G. Angenneister, Terr. Magn., 25, 26-32, (1920).

(5) H. Hatakeyama, Geophys. Magazine, 12, 173.188, 189-210, (1938).

${ }^{(6)}$ P. Giacomo, Ann. de Géophysique, 5, 171-173, (1949).

(7) T. Terada, Journ. of the Coll. of. Sc., Imp. Univ., XXVII, 9, Tokyo, (1917).

$\left(^{8}\right)$ Y. Kato, J. Ossaka, M. OKuda, The Sc. Rep. of the Tohoku Univ., Ser. 5, Geophysics, 5, 1-21, (1953).

$\left({ }^{9}\right)$ J. G. Scholte, Veldkamp, J. Atm. Terr. P'hys. 6, 33-45, (1955).

$\left({ }^{10}\right)$ Y. Kato, K. Ossaka, T. Watanabe, M. Okuda, T. Tamao, The Sc. Rep. of the Tohoku Univ. Ser. 5, Geophysics, 7, 136-146, (1956).

(11) Y. Като, J. Ossaks, The Sc. Rep. of the Tohoku Univ. Ser. 5, Geophysics, 4, 61-63, (1952). Voir aussi G. Grenet, Y. Kato, J. Ossaka, M. OKUDA, ibid., 6, 1-10, (1954).

(12) K. Yanaginara, Memoirs of the Kakioka Magnetie Observatory, 8, 61-76, (1957).

${ }^{(13)}$ Kato, Ossaka, The Se. Rep. of the Tohoku University, Series 5, Geophysics, 3, 111-113, (1951).

(14) Citernosky, Coon, Maple, Nat. Res. Couneil, Ann. Geophys. Union 33d Annual Meeting, 320.

(15) G. Billaud, Travaux de l'Inst. de Mét. et de Phys. du Gl. de l'Algerie, 9, 12-49, (1953). - J. L. Bureau, Ibid., 15, 1-32, (1956); les années 1955 à 1957 nous ont été communiquées gracieusement.

${ }^{(16)}$ P. Rougerie, Ann. de Géophysique, 10, fig. 2, 50, (1954). 一 O. MEYER, Deutsche Hydrographische Zeitschrift, 4, 61-65, (1951).

(17) K. YanaginaRA, Memoirs of the Kalioka Magnetic Observatory, 8, 1, 49-59, (1957).

(18) G. Grenet, Travaux de l'Institut de Météorologie et Physique du Globe de l'Algérie, 9, 54-55, (1953).

${ }^{(19)}$ G. Kunetz, Exposé au séminaire de Physique du Globe, Paris 1956, non publié.

(20) M. Schlumberger et G. Kunetz, Communication présentée à l'Assem. blée Générale de l'U.G.G.I., Oslo 948.

(21) G. Angenieister, Gerl. Beitr. z. Geophysik, 64, 108-132, (1954).

(22) J. UnTIEDT, Zeitschrift für Geophysik, 24, 125-133, (1958). 
(29) J. Coulomв, Ann. de Géophysique, 10, 159-161, (1954).

$\left.{ }^{24}\right)$ G. Kunetz, H. Richard, Atti del VII Convegno Nazionale del Metano et del Petrolio, Taormina, 1952. - G. Kunetz, Ann. de Géophysique, 10, 262-270, (1954).

${ }^{\left({ }^{25}\right)}$ Y. Kato, M. OKuda, Sc. Rep. of the Toholu Univ., Ser. 5, 7, Suppl., 37 41, (1956).

${ }^{(26)}$ J. L. Bureau, Comptes-rendus Ac. Se., 247, 112-114, (1958).

${ }^{(23)}$ Y. Kато, T. SAITO, The Se. Rep. of the Tohoku University, Ser. 5, Geophysics, 9, 99-105, (1958).

$\left({ }^{28}\right)$ L. Harang, Terr. Magn., 41, 329, (1946); 44, 17, (1959).

${ }^{29}$ E. SucksdorfF, Terr. Magn., 44, 157, (1944). 